\title{
Accuracy of the Kato-Katz method and formalin-ether concentration technique for the diagnosis of Clonorchis sinensis, and implication for assessing drug efficacy
}

Men-Bao Qian ${ }^{1}$, Peiling Yap ${ }^{2,3}$, Yi-Chao Yang ${ }^{4}$, Hai Liang ${ }^{5}$, Zhi-Hua Jiang ${ }^{4}$, Wei Li ${ }^{5}$, Jürg Utzinger ${ }^{2,3}$, Xiao-Nong Zhou' ${ }^{1}$ and Jennifer Keiser ${ }^{3,6^{*}}$

\begin{abstract}
Background: Clonorchiasis is a chronic neglected disease caused by a liver fluke, Clonorchis sinensis. Chemotherapy is the mainstay of control and treatment efficacy is usually determined by microscopic examination of fecal samples. We assessed the diagnostic accuracy of the Kato-Katz method and the formalin-ether concentration technique (FECT) for $C$. sinensis diagnosis, and studied the effect of diagnostic approach on drug efficacy evaluation.

Methods: Overall, 74 individuals aged $\geq 18$ years with a parasitological confirmed $C$. sinensis infection at baseline were re-examined 3 weeks after treatment. Before and after treatment, two stool samples were obtained from each participant and each sample was subjected to triplicate Kato-Katz thick smears and a single FECT examination.

Results: Thirty-eight individuals were still positive for C. sinensis according to our diagnostic 'gold' standard (six Kato-Katz thick smears plus two FECT). Two FECT had a significantly lower sensitivity than six Kato-Katz thick smears (44.7\% versus 92.1\%; $p<0.001$ ). Examination of single Kato-Katz and single FECT considerably overestimated cure rates.
\end{abstract}

Conclusions: In settings where molecular diagnostic assays are absent, multiple Kato-Katz thick smears should be examined for an accurate diagnosis of $C$. sinensis infection and for assessing drug efficacy against this liver fluke infection.

Keywords: Clonorchis sinensis, Kato-Katz method, Formalin-ether concentration technique, Diagnosis, Efficacy

\section{Background}

Clonorchiasis is a chronic neglected disease caused by consumption of raw or undercooked freshwater fish harboring the infective metacercariae of a liver fluke, Clonorchis sinensis. An estimated 15 million people are infected worldwide, but the current hotspots of infection are concentrated in the People's Republic of China (P.R. China), the Republic of Korea and Vietnam [1-7]. Chronic C. sinensis infection is associated with liver and biliary conditions, such as gallstone, cholecystitis,

\footnotetext{
* Correspondence: jennifer.keiser@unibas.ch

${ }^{3}$ University of Basel, Basel, Switzerland

${ }^{6}$ Department of Medical Parasitology and Infection Biology, Swiss Tropical and Public Health Institute, Basel, Switzerland

Full list of author information is available at the end of the article
}

cholangitis and cholangiocarcinoma (CCA) [1,3,6,8-11]. CCA is a carcinoma arising in any part of the biliary tree with poor prognosis, causally linked to an infection with C. sinensis [12,13], and it has been estimated that several thousand new CCA cases occur annually due to this liver fluke infection $[3,6,14]$.

Chemotherapy with praziquantel is the main strategy to control morbidity due to clonorchiasis [15-17]. Praziquantel is a safe and efficacious drug, usually resulting in high cure and egg reduction rates [18-20]. However, adverse events, including dizziness, sleepiness, headache and diarrhea, following praziquantel administration have been observed, and they might compromise patient compliance. Furthermore, taking into consideration the largescale use of praziquantel, particularly in the frame of 
large-scale chemotherapy targeting schistosomiasis [21], there is concern that parasites might develop resistance against praziquantel. The development of new drugs against clonorchiasis and other chronic neglected diseases is thus of high priority [20].

The diagnosis of helminth infections is largely dependent on fecal examination and the most widely used methods are the Kato-Katz technique [22] and the formalin-ether concentration technique (FECT) [23]. The Kato-Katz technique is commonly employed in field surveys due to the relative ease of operation and the possibility to quantify infection intensity, which allows stratification into different intensity classes based on cut-offs provided by the World Health Organization (WHO) [24]. On the other hand, FECT is usually conducted in specialized laboratories for the concurrent diagnosis of helminth and intestinal protozoan infections [25-27].

The aim of this study was to comparatively assess the accuracy of Kato-Katz and FECT for the diagnosis of C. sinensis, and to investigate the effect of the diagnostic technique and sampling effort on estimating drug efficacy. Recently, tribendimidine, a drug registered in P.R. China for treating soil-transmitted helminth infection [28], was tested against C. sinensis infection in an exploratory clinical trial [29]. Our study was integrated into this trial, which assessed the efficacy of three different treatment regimens; (i) praziquantel $(75 \mathrm{mg} / \mathrm{kg}$ divided into three doses); (ii) single-dose tribendimidine (400 $\mathrm{mg}$ ); and (iii) triple-dose tribendimidine (400 mg daily for three days) [29].

\section{Methods}

\section{Ethical considerations}

Our study was approved by the ethics committees in Basel, Switzerland (EKBB; reference nos. 209/09 and 375/ 11), the Liverpool School of Tropical Medicine (Liverpool, UK; reference no. 12.02RS), and the National Institute of Parasitic Diseases, Chinese Center for Disease Control and Prevention (Shanghai, P.R. China; reference no. 2012-02). A written informed consent form was obtained from each participant. The trial is registered with Current Controlled Trials (identifier: ISRCTN80829842).

\section{Study participants and sample collection}

The study was carried out in the township of Gantang, Binyang county, Guangxi Zhuang Autonomous Region, P.R. China, between June and July 2012. Seventy-four individuals aged 18 years and above with a parasitological confirmed C. sinensis infection were included. Before treatment, each participant provided two fresh morning stool samples within 5 days. Three weeks after treatment (praziquantel $75 \mathrm{mg} / \mathrm{kg}$ given in three dosages and tribendimidine single- or triple-dose, $400 \mathrm{mg}$ each), two stool samples were again collected from each participant. Sample size calculation, inclusion/exclusion criteria and randomization procedure have been described elsewhere [29].

\section{Laboratory procedures}

Triplicate Kato-Katz thick smears, using standard $41.7 \mathrm{mg}$ templates, were prepared from each stool sample. The Kato-Katz thick smears were examined under a microscope by experienced technicians and the number of $C$. sinensis eggs counted and recorded. Additionally, approximately $1 \mathrm{~g}$ of stool from each sample was kept in $10 \mathrm{ml}$ sodium acetate-acetic acid-formalin (SAF) solution, thoroughly broken up and emulsified for subsequent FECT examination [26,27]. Briefly, the SAF solution was composed of $15 \mathrm{~g}$ sodium acetate, $20 \mathrm{ml}$ acetic acid and

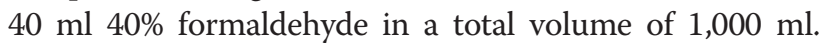
For microscopic examination, the fixed sample was first re-suspended and strained through a medical gauze into a conical tube. After centrifugation for $1 \mathrm{~min}$ at $500 \times \mathrm{g}$, the supernatant was decanted. If the final sediment contained a volume of more than $1 \mathrm{ml}$, the first two steps were repeated and part of the suspension removed. Subsequently, $7 \mathrm{ml} 0.85 \%$ sodium chloride and $2-3 \mathrm{ml}$ diethyl ether were added to the remaining sediment. The tube was closed with a rubber stopper, shaken vigorously $(\sim 30 \mathrm{sec})$ and centrifuged for $5 \mathrm{~min}$ at $500 \times \mathrm{g}$. From the resulting four layers, the first three layers were removed and the remaining layer examined for $C$. sinensis eggs. The number of $C$. sinensis eggs was counted and recorded. When more than 100 eggs for $C$. sinensis were encountered, the laboratory technician noted '100+'.

\section{Statistical analysis}

We used STATA version 10.0 (Stata Corp.; College Station, TX, USA) for statistical analysis. As diagnostic 'gold' standard, we defined a combination of six Kato-Katz thick smears plus two FECT (two stool samples each subjected to triplicate Kato-Katz thick smears and single FECT). Prevalence, infection intensity (determined by the geometric mean of eggs per $1 \mathrm{~g}$ of stool (GM EPG)) and cure rate $(\mathrm{CR})$ for different treatment arms, as assessed by different diagnostic methods and efforts (i.e. single Kato-Katz, triplicate Kato-Katz and single FECT from first stool sample; six Kato-Katz and two FECT), were compared. CR was determined as the proportion of C. sinensis egg-positive individuals at baseline, who became egg-negative 3 weeks after treatment. Sensitivity was calculated and presented with a 95\% confidence interval (CI). Difference in sensitivity between three or six KatoKatz thick smears and a single or duplicate FECT was determined by McNemar test on positive individuals. Finally, the agreement between three or six Kato-Katz thick smears and single or duplicate FECT for detecting $C$. sinensis positive or negative individuals, was 
assessed using Kappa $(\kappa)$ statistic with the following cutoffs: $\mathrm{\kappa}<0$, no agreement; $\mathrm{\kappa}=0.01-0.2$, poor agreement; $\mathrm{\kappa}=$ 0.21-0.4, fair agreement; $\kappa=0.41-0.6$, moderate agreement; $\mathrm{K}=0.61-0.8$, substantial agreement; $\mathrm{K}=0.81-1.0$, almost perfect agreement $[27,30,31]$.

\section{Results}

\section{Diagnostic accuracy}

At the pre-treatment survey, 74 individuals were diagnosed with C. sinensis eggs in their stool regardless of the diagnostic technique used (sensitivity for both methods: $100 \%)$. Baseline infection intensities obtained with KatoKatz through different sampling efforts ranged from 2,229 (first Kato-Katz thick smear) to 3,390 GM EPG (six Kato-
Katz thick smears). Semi-quantification of infection intensity determined with FECT examination was possible and the results are shown in Table 1.

Three weeks post-treatment, a total of 38 individuals (51.4\%) were diagnosed with C. sinensis eggs in their stool, according to our diagnostic 'gold' standard. Among them, $35(47.3 \%)$ were found to be positive when analyzing six Kato-Katz thick smears, which was more than double the number compared to duplicate FECT (17 positive; $23.0 \% ; p=0.001)$. The number of positives detected with the first Kato-Katz, triplicate Kato-Katz or single FECT on the first stool sample were 23 (31.1\%), 29 (39.2\%) and 13 (17.6\%), respectively. Six Kato-Katz thick smears detected C. sinensis infections with a significantly higher sensitivity

Table 1 Accuracy of Kato-Katz method (different sampling efforts) and formalin-ether concentration technique (FECT) for the diagnosis of Clonorchis sinensis at baseline and $\mathbf{3}$ weeks after treating $\mathbf{7 4}$ individuals with a parasitological confirmed infection

\begin{tabular}{|c|c|c|c|c|c|c|c|c|c|}
\hline \multirow{3}{*}{$\begin{array}{l}\text { Diagnostic } \\
\text { approach }\end{array}$} & \multirow{3}{*}{$\begin{array}{l}\text { Infection } \\
\text { intensity }\end{array}$} & \multicolumn{3}{|c|}{ Positives at baseline } & \multicolumn{4}{|c|}{ Positives at 3 week post-treatment follow-up } & \multirow{3}{*}{$\begin{array}{c}\text { Sensitivity } \\
{[\%(95 \% \mathrm{CI})]}\end{array}$} \\
\hline & & \multirow[t]{2}{*}{ No. } & \multirow[t]{2}{*}{$\%$} & \multirow{2}{*}{$\begin{array}{l}\text { Infection intensity } \\
\text { [EPG (range)] }^{\mathrm{a}}\end{array}$} & \multirow[t]{2}{*}{ No. } & \multirow[t]{2}{*}{$\%(95 \% \mathrm{Cl})$} & \multicolumn{2}{|c|}{ Infection intensity [EPG (range)] } & \\
\hline & & & & & & & Positive & False negative & \\
\hline \multirow[t]{4}{*}{ Single Kato-Katz } & Light & 22 & 29.7 & $266(24-960)$ & 20 & $27.0(17.4-38.6)$ & $89(24-696)$ & n.a & n.a \\
\hline & Moderate & 35 & 47.3 & $2,952(1,080-8,976)$ & 3 & $4.1(0.8-11.4)$ & $2,525(1,512-4,224)$ & n.a & n.a \\
\hline & Heavy & 17 & 23.0 & $19,555(10,080-47,376)$ & 0 & 0 & n.a & n.a & n.a \\
\hline & Overall & 74 & 100.0 & $2,229(24-47,376)$ & 23 & $31.1(20.8-42.9)$ & $138(24-4,224)$ & $17.6(4-72)^{c}$ & $60.5(43.5-75.5)$ \\
\hline \multirow[t]{4}{*}{ Triplicate Kato-Katz } & Light & 17 & 23.0 & $295(80-864)$ & 26 & $35.1(24.4-47.1)$ & $60(8-760)$ & n.a & n.a \\
\hline & Moderate & 38 & 51.4 & $2,889(1,024-9,992)$ & 3 & $4.1(0.8-11.4)$ & $2,482(1,984-3,136)$ & n.a & n.a \\
\hline & Heavy & 19 & 25.7 & $18,390(10,312-52,240)$ & 0 & 0 & n.a & n.a & n.a \\
\hline & Overall & 74 & 100.0 & $2,750(80-52,240)$ & 29 & $39.2(28.0-51.2)$ & $88(8-3,136)$ & $16.3(4-72)^{c}$ & $76.3(59.4-88.0)$ \\
\hline \multirow[t]{4}{*}{ Six Kato-Katz } & Light & 13 & 17.6 & $350(124-788)$ & 31 & $41.9(30.5-53.9)$ & $35(4-704)$ & n.a & n.a \\
\hline & Moderate & 41 & 55.4 & $3,050(1,032-9,428)$ & 4 & $5.4(1.5-13.3)$ & $1,808(1,208-3,636)$ & n.a & n.a \\
\hline & Heavy & 20 & 27.0 & $18,426(10,656-31,244)$ & 0 & 0 & n.a & n.a & n.a \\
\hline & Overall & 74 & 100.0 & $3390(124-31,244)$ & 35 & $47.3(35.6-59.3)$ & $55(4-3,636)$ & n.a & $92.1(77.5-97.9)$ \\
\hline \multirow[t]{4}{*}{ Single FECT ${ }^{d}$} & Light & 51 & 68.9 & $11.7(1-100)$ & 13 & $17.6(9.7-28.2)$ & $3.6(1-21)$ & n.a & n.a \\
\hline & Moderate & 0 & 0 & n.a & 0 & 0 & n.a & n.a & n.a \\
\hline & Heavy & 23 & 31.1 & n.a & 0 & 0 & n.a & n.a & n.a \\
\hline & Overall & 74 & 100.0 & n.a & 13 & $17.6(9.7-28.2)$ & $3.6(1-21)$ & $0.7(0.5-1)^{e}$ & $34.2(20.1-51.4)^{f, g}$ \\
\hline \multirow[t]{4}{*}{ Duplicate FECT ${ }^{\mathrm{h}}$} & Light & 35 & 47.3 & $19.2(2-62)$ & 17 & $23.0(14.0-43.2)$ & $1.7(0.5-14.5)$ & n.a & n.a \\
\hline & Moderate & 22 & 29.7 & n.a & 0 & 0 & n.a & n.a & n.a \\
\hline & Heavy & 17 & 23.0 & n.a & 0 & 0 & n.a & n.a & n.a \\
\hline & Overall & 74 & 100.0 & n.a & 17 & $23.0(14.0-43.2)$ & $1.7(0.5-14.5)$ & n.a & $44.7(29.0-61.5)^{\mathrm{i}, j}$ \\
\hline 'Gold' standard'k & n.a & 74 & 100.0 & n.a & 38 & $51.4(39.4-63.1)$ & n.a & n.a & 100.0 \\
\hline
\end{tabular}

$\mathrm{Cl}$, confidence interval; EPG, eggs per $1 \mathrm{~g}$ of stool; n.a., not applicable.

${ }^{a} E P G$ is presented as geometric mean.

bLight infection, 1-999 EPG; moderate infection, 1,000-9,999 EPG; heavy infection: $\geq 10,000$ EPG.

'EPG determined by six Kato-Katz thick smears.

dLight infection: $\leq 100$ EPG; heavy infection: >100 EPG.

EEP determined by duplicate FECT.

fDifference in sensitivities between three Kato-Katz and single FECT determined by the McNemar test on positive individuals: $p<0.001$.

${ }^{9} \mathrm{~K}$ measure of agreement between three Kato-Katz and single FECT taking into account positive and negative individuals: 0.37 .

hLight infection: both samples $\leq 100$ EPG; moderate infection: 1 sample $\leq 100$ EPG and 1 sample >100 EPG; heavy infection: both samples >100 EPG.

'Difference in sensitivities between six Kato-Katz and two FECT determined by the McNemar test on positive individuals: $p<0.001$.

${ }^{\mathrm{j}} \mathrm{K}$ measure of agreement between six Kato-Katz and two FECT taking into account positive and negative individuals: 0.33 .

kDiagnostic 'gold' standard is defined as a combination of six Kato-Katz and duplicate FECT. 
than duplicate FECT (92.1\% versus $44.7 \%$; $p<0.001)$. Similarly, triplicate Kato-Katz was significantly more sensitive than single FECT on the first stool sample $(76.3 \%$ versus $34.2 \%$; $p$ 0.001). There was poor agreement between six Kato-Katz thick smears and duplicate FECT $(\kappa=0.33)$, as well as triplicate Kato-Katz thick smears and single FECT on the first stool sample $(\kappa=0.37)$. Post-treatment infection intensities obtained with Kato-Katz through different sampling efforts ranged from 138 (first Kato-Katz thick smear) to 55 GM EPG (six Kato-Katz thick smears). On the other hand, the 13 and 17 positives detected by single and duplicate FECT had infection intensities of 3.6 and 1.7 GM EPG, respectively.

Patients missed by single and triplicate Kato-Katz thick smears but identified as positive according to six Kato-Katz thick smears had infection intensities (based on six Kato-Katz) of 17.6 and 16.3 GM EPG, respectively. Four patients that were negative according to a single FECT had a GM EPG of 0.7 based on duplicate FECT.

\section{Effect of diagnostic approach on treatment efficacy}

Table 2 shows the treatment efficacy, as determined by the observed CR, in relation to the diagnostic approach taken. According to our diagnostic 'gold' standard (six Kato-Katz plus duplicate FECT), overall CRs of $44 \%$ (single-dose tribendimidine) to 52\% (praziquantel) were achieved. Similar CRs were observed with six Kato-Katz thick smears, whereas a single Kato-Katz or FECT resulted in considerably higher CRs. However, no statistically significant differences in the efficacy between praziquantel, tribendimidine single- and triple-dose were observed against $C$. sinensis infection regardless of the diagnostic approach taken.

\section{Discussion}

The control of clonorchiasis and other food-borne trematodiases has gained attention in recent years due to the growing recognition of the public health importance of these chronic parasitic diseases $[3,5,17,32]$. Chemotherapy is the intervention of choice for morbidity control against clonorchiasis $[17,32]$, as well as other helminthiases [33].

To evaluate anthelmintic drug efficacy, an accurate diagnosis is important [34]. However, the sensitivity of diagnostic methods varies, and it is particularly challenging to identify low-intensity helminth infection with high accuracy [27]. In the present study, we compared the accuracy of the widely used Kato-Katz method (different sampling efforts) with FECT for the diagnosis of C. sinensis infection within the frame of a clinical trial. Of note, while the Kato-Katz method was initially developed for the diagnosis of intestinal schistosomiasis [22], it is now routinely used for a wide variety of helminth infections [35], including C. sinensis and other fluke infections [36]. In our study, two stool samples were collected at the 3-week post-treatment follow-up from all participants who had a parasitological confirmed C. sinensis infection at the baseline survey and had undergone treatment with praziquantel or tribendimidine. The number of individuals identified with $C$. sinensis eggs in their stool after treatment varied considerably as a result of the diagnostic approach used. For example, while a single FECT was able to diagnose all positive cases at baseline (sensitivity: 100\%), at the post-treatment follow-up, when low-intensity infections were common, FECT only diagnosed 13 (single FECT) and 17 (duplicate FECT) positive cases, respectively, whereas a total of 35 C. sinensis-positive cases were detected based on six Kato-Katz thick smears. Consequently, the sensitivity of FECT at post-treatment was below 50\%, whereas six KatoKatz showed a high sensitivity (92.1\%). In addition, FECT examination only allows recording $C$. sinensis eggs in a semi-quantitative manner. The inferiority of FECT compared to the Kato-Katz method reported here corroborates recent results from other studies on another liver fluke infection. Indeed, Soukhathammavong and colleagues [37] and Lovis and collaborators [38] reported that the KatoKatz method is considerably more sensitive than FECT in

Table 2 Estimated cure rates of three different treatment regimens against Clonorchis sinensis infection, according to different diagnostic approaches

\begin{tabular}{|c|c|c|c|}
\hline \multirow[t]{3}{*}{ Diagnostic approach } & \multicolumn{3}{|c|}{ Observed cure rate $[\%(95 \% \mathrm{Cl})]$} \\
\hline & Praziquantel & Tribendimidine & Tribendimidine \\
\hline & (75 mg/kg divided in 3 doses) & (400 mg once daily for 3 days) & (400 mg once) \\
\hline Single Kato-Katz & $72.0(50.6-87.9)$ & $75.0(53.3-90.2)$ & $60.0(38.7-78.9)$ \\
\hline Triplicate Kato-Katz & $60.0(38.7-78.9)$ & $66.7(44.7-84.4)$ & $56.0(34.9-75.6)$ \\
\hline Six Kato-Katz & $56.0(34.9-75.6)$ & $58.3(36.6-77.9)$ & $44.0(24.4-65.1)$ \\
\hline Single FECT & $84.0(63.9-95.5)$ & $87.5(67.6-97.3)$ & $76.0(54.9-90.6)$ \\
\hline Duplicate FECT & $80.0(59.3-93.2)$ & $79.2(57.8-92.9)$ & $72.0(50.6-87.9)$ \\
\hline Diagnostic 'gold' standard ${ }^{a}$ & $52.0(31.3-72.2)$ & $50.0(29.1-70.9)$ & $44.0(24.4-65.1)$ \\
\hline
\end{tabular}

$\mathrm{Cl}$, confidence interval.

a'Diagnostic 'gold' standard is defined as a combination of six Kato-Katz and duplicate FECT. 
detecting Opisthorchis viverrini infection. Prior work focusing on the blood fluke Schistosoma mansoni speculated that the multiple washing steps in the FECT could influence its sensitivity [39]. Loss of eggs at washing steps might also explain the low EPGs observed with FECT when compared to the Kato-Katz method. However, the use of dyes to stain eggs examined with FECT would allow differential diagnosis of liver and intestinal flukes, which co-exist in our and other study areas in Southeast Asia $[40,41]$.

Our findings have important ramifications on assessing treatment efficacy. Using the insensitive FECT would result in considerably overestimated treatment efficacy against C. sinensis. Indeed, the reported CRs using duplicate FECT were 72.0-80.0\%, whereas six Kato-Katz showed considerably lower CRs (44.0-58.3\%). Hence, the diagnostic approach should be taken into consideration when reporting and interpreting treatment efficacies.

\section{Conclusions}

The Kato-Katz method is more reliable than FECT for the diagnosis of $C$. sinensis and for evaluating drug efficacy (as determined by $\mathrm{CR}$ ) against this liver fluke infection, particularly when multiple thick smears are analyzed. Although collection of more than one stool sample causes logistic inconvenience and might lower patient compliance, we recommend that at least two stool samples should be obtained in order to achieve an accurate diagnosis at treatment follow-up. FECT cannot be recommended for the diagnosis of clonorchiasis unless it is combined with other techniques, such as polymerase chain reaction (PCR), a highly sensitive approach, which is gaining importance for helminth diagnosis [42-44], but might not be available in resource-constrained settings.

\section{Competing interests}

The authors declare that they have no competing interests.

\section{Authors' contributions \\ Conceived and designed the experiments: JU, XNZ, JK. Performed the experiments: $M B Q, P Y, Y C Y, H L, Z H J, W L, J K$. Analyzed the data: $M B Q, P Y$. Contributed reagents/materials/analysis tools: $\mathrm{MBQ}, \mathrm{PY}, \mathrm{YCY}, \mathrm{HL}$. Wrote the paper: MBQ, PY, JU, JK. All authors read and approved the final version of the manuscript.}

\section{Acknowledgments}

We are indebted to the local staff of Guangxi CDC and Binyang CDC for field organization, sample collection and microscopic examination. This work was supported by DFID/MRC/Wellcome Trust Joint Global Health Trials Scheme. JK is financially supported by the Swiss National Science Foundation (SNSF; projects nos. PPOOA-114941 and PPOOP3_135170). The funders (DFID/MRC/Wellcome Trust Joint Global Health Trials Scheme and SNSF) had no role in study design, data collection and analysis, decision to publish, nor preparation of the manuscript.

\section{Author details}

'National Institute of Parasitic Diseases, Chinese Center for Disease Control and Prevention, WHO Collaborative Center for Malaria, Schistosomiasis and Filariasis, Key Laboratory of Parasite and Vector Biology, Ministry of Health, Shanghai, People's Republic of China. ${ }^{2}$ Department of Epidemiology and Public Health, Swiss Tropical and Public Health Institute, Basel, Switzerland. ${ }^{3}$ University of Basel, Basel, Switzerland. ${ }^{4}$ Guangxi Center for Disease Control and Prevention, Nanning, People's Republic of China. ${ }^{5}$ Binyang Center for Disease Control and Prevention, Binyang, People's Republic of China. ${ }^{6}$ Department of Medical Parasitology and Infection Biology, Swiss Tropical and Public Health Institute, Basel, Switzerland.

Received: 3 September 2013 Accepted: 23 October 2013 Published: 29 October 2013

\section{References}

1. Lun ZR, Gasser RB, Lai DH, Li AX, Zhu XQ, Yu XB, Fang YY: Clonorchiasis: a key foodborne zoonosis in China. Lancet Infect Dis 2005, 5:31-41.

2. Shin EH, Guk SM, Kim HJ, Lee SH, Chai JY: Trends in parasitic diseases in the Republic of Korea. Trends Parasitol 2008, 24:143-150.

3. Fürst T, Keiser J, Utzinger J: Global burden of human food-borne trematodiasis: a systematic review and meta-analysis. Lancet Infect Dis 2012, 12:210-221.

4. Fürst T, Duthaler U, Sripa B, Utzinger J, Keiser J: Trematode infections: liver and lung flukes. Infect Dis Clin North Am 2012, 26:399-419.

5. Hong ST, Fang Y: Clonorchis sinensis and clonorchiasis, an update. Parasitol Int 2012, 61:17-24.

6. Qian MB, Chen YD, Liang S, Yang GJ, Zhou XN: The global epidemiology of clonorchiasis and its relation with cholangiocarcinoma. Infect Dis Poverty 2012, 1:4.

7. Qian MB, Chen YD, Yan F: Time to tackle clonorchiasis in China. Infect Dis Poverty 2013, 2:4.

8. Bouvard V, Baan R, Straif K, Grosse Y, Secretan B, El Ghissassi F, BenbrahimTallaa L, Guha N, Freeman C, Galichet L, Cogliano V: A review of human carcinogens-part B: biological agents. Lancet Oncol 2009, 10:321-322.

9. Sripa B, Kaewkes S, Intapan PM, Maleewong W, Brindley PJ: Food-borne trematodiases in Southeast Asia: epidemiology, pathology, clinical manifestation and control. Adv Parasitol 2010, 72:305-350.

10. Qian MB, Chen YD, Fang YY, Xu LQ, Zhu TJ, Tan T, Zhou CH, Wang GF, Jia TW, Yang GJ, Zhou XN: Disability weight of Clonorchis sinensis infection: captured from community study and model simulation. PLoS Negl Trop Dis 2011, 5:e1377.

11. Fürst $T$, Sayasone $S$, Odermatt $P$, Keiser J, Utzinger J: Manifestation, diagnosis, and management of foodborne trematodiasis. BMJ 2012, 344:e4093.

12. Khan SA, Toledano MB, Taylor-Robinson SD: Epidemiology, risk factors, and pathogenesis of cholangiocarcinoma. HPB (Oxford) 2008, 10:77-82.

13. IARC: Biological agents: a review of human carcinogens. Monographs on the evaluation of carcinogenic risks to humans. Lyon: International Agency for Research on Cancer; 2012.

14. Parkin DM: The global health burden of infection-associated cancers in the year 2002. Int J Cancer 2006, 118:3030-3044.

15. Montresor A, Cong DT, Sinuon M, Tsuyuoka R, Chanthavisouk C, Strandgaard H, Velayudhan R, Capuano CM, Le Anh T, Tee Dato AS: Large-scale preventive chemotherapy for the control of helminth infection in Western Pacific countries: six years later. PLoS Negl Trop Dis 2008, 2:e278.

16. Choi MH, Park SK, Li Z, Ji Z, Yu G, Feng Z, Xu L, Cho SY, Rim HJ, Lee SH, Hong ST: Effect of control strategies on prevalence, incidence and reinfection of clonorchiasis in endemic areas of China. PLoS Negl Trop Dis 2010, 4:e601.

17. WHO: Working to overcome the global impact of ngelected tropical diseases: first WHO report on neglected tropical diseases. Geneva: World Health Organization; 2010.

18. Seo BS, Lee SH, Chai JY, Hong ST: Praziquantel (Distocide ${ }^{\oplus}$ ) in treatment of Clonorchis sinensis infection. Korean J Parasitol 1983, 21:241-245.

19. Chen MG, Hua XJ, Wan ZR, Weng YQ, Wang MJ, Zhu PJ, He BZ, Xu MY: Praziquantel in 237 cases of Clonorchis sinensis. Chin Med J (Engl) 1983, 96:935-940.

20. Keiser J, Utzinger J: The drugs we have and the drugs we need against major helminth infections. Adv Parasitol 2010, 73:197-230.

21. WHO: Schistosomiasis: number of people treated in 2011. Wkly Epidemiol Rec 2013, 88:81-88.

22. Katz N, Chaves A, Pellegrino J: A simple device for quantitative stool thicksmear technique in schistosomiasis mansoni. Rev Inst Med Trop São Paulo 1972, 14:397-400. 
23. Johansen MV, Sithithaworn P, Bergquist R, Utzinger J: Towards improved diagnosis of zoonotic trematode infections in Southeast Asia. Adv Parasito 2010, 73:171-195.

24. WHO: Prevention and control of schistosomiasis and soil-transmitted helminthiasis: report of a WHO expert committee. WHO Tech Rep Ser 2002, 912:1-57.

25. Allen AVH, Ridley DS: Further observations on the formol-ether concentration technique for faecal parasites. J Clin Pathol 1970, 23:545-546.

26. Marti H: SAF-an alternative fixation solution for parasitological stool specimens. Schweiz Med Wochenschr 1990, 120:1473-1476.

27. Utzinger J, Botero-Kleiven S, Castelli F, Chiodini PL, Edwards H, Kohler N, Gulletta M, Lebbad M, Manser M, Matthys B, N'Goran EK, Tannich E, Vounatsou P, Marti H: Microscopic diagnosis of sodium acetate-acetic acid-formalin-fixed stool samples for helminths and intestinal protozoa: a comparison among European reference laboratories. Clin Microbiol Infect 2010, 16:267-273.

28. Xiao SH, Utzinger J, Tanner M, Keiser J, Xue J: Advances with the Chinese anthelminthic drug tribendimidine in clinical trials and laboratory investigations. Acta Trop 2013, 126:115-126

29. Qian MB, Yap P, Yang YC, Liang H, Jiang ZH, Li W, Tan YG, Zhou H, Utzinger J, Zhou XN, Keiser J: Efficacy and safety of tribendimidine against Clonorchis sinensis. Clin Infect Dis 2013, 56:e76-e82.

30. Cohen J: A coefficient of agreement for nominal scales. Educ Psychol Meas 1960, 20:37-46.

31. Landis JR, Koch GG: The measurement of observer agreement for categorical data. Biometrics 1977, 33:159-174.

32. Ministry of Health: Control program for important parasitic diseases between 2006 and 2015. Available at: http://www.moh.gov.cn/mohbgt/pw10604/ 200804/27592.shtml (accessed: 18 October 2013).

33. Hotez PJ, Molyneux DH, Fenwick A, Kumaresan J, Ehrlich Sachs S, Sachs JD, Savioli L: Control of neglected tropical diseases. N Engl J Med 2007, 357:1018-1027.

34. Knopp S, Speich B, Hattendorf J, Rinaldi L, Mohammed KA, Khamis IS, Mohammed AS, Albonico M, Rollinson D, Marti H, Cringoli G, Utzinger J: Diagnostic accuracy of Kato-Katz and FLOTAC for assessing anthelmintic drug efficacy. PLoS Negl Trop Dis 2011, 5:e1036.

35. Knopp S, Steinmann P, Keiser J, Utzinger J: Nematode infections: soiltransmitted helminths and Trichinella. Infect Dis Clin North Am 2012 26:341-358

36. Chen YD, Zhou CH, Xu LQ: Analysis of the results of two nationwide surveys on Clonorchis sinensis infection in China. Biomed Environ Sci 2012, 25:163-166.

37. Soukhathammavong $P$, Odermatt $P$, Sayasone $S$, Vonghachack $Y$, Vounatsou P, Hatz C, Akkhavong K, Keiser J: Efficacy and safety of mefloquine, artesunate, mefloquine-artesunate, tribendimidine, and praziquantel in patients with Opisthorchis viverrini: a randomised, exploratory, openlabel, phase 2 trial. Lancet Infect Dis 2011, 11:110-118.

38. Lovis L, Mak TK, Phongluxa K, Aye Soukhathammavong P, Vonghachack Y, Keiser J, Vounatsou P, Tanner M, Hatz C, Utzinger J, Odermatt P, Akkhavong K: Efficacy of praziquantel against Schistosoma mekongi and Opisthorchis viverrini: a randomized, single-blinded dose-comparison trial. PLoS Negl Trop Dis 2012, 6:e1726.

39. Ebrahim A, El-Morshedy H, Omer E, El-Daly S, Barakat R: Evaluation of the Kato-Katz thick smear and formol ether sedimentation techniques for quantitative diagnosis of Schistosoma mansoni infection. Am J Trop Med Hyg 1997, 57:706-708.

40. Lovis L, Mak TK, Phongluxa K, Soukhathammavong P, Sayasone S, Akkhavong K, Odermatt P, Keiser J, Felger I: PCR diagnosis of Opisthorchis viverrini and Haplorchis taichui infections in a Lao community in an area of endemicity and comparison of diagnostic methods for parasitological field surveys. J Clin Microbiol 2009, 47:1517-1523.

41. Jeon HK, Lee D, Park H, Min DY, Rim HJ, Zhang H, Yang Y, Li X, Eom KS: Human infections with liver and minute intestinal flukes in Guangxi, China: analysis by DNA sequencing, ultrasonography, and immunoaffinity chromatography. Korean J Parasitol 2012, 50:391-394.

42. Knopp S, Mgeni AF, Khamis IS, Steinmann P, Stothard JR, Rollinson D, Marti H, Utzinger J: Diagnosis of soil-transmitted helminths in the era of preventive chemotherapy: effect of multiple stool sampling and use of different diagnostic techniques. PLoS Negl Trop Dis 2008, 2:e331.

43. Steinmann P, Du ZW, Wang LB, Wang XZ, Jiang JY, Li LH, Marti H, Zhou XN, Utzinger J: Extensive multiparasitism in a village of Yunnan province,
People's Republic of China, revealed by a suite of diagnostic methods. Am J Trop Med Hyg 2008, 78:760-769.

44. Cho PY, Na BK, Choi KM, Kim JS, Cho SH, Lee WJ, Lim SB, Cha SH, Park YK, Pak JH, Lee HW, Hong SJ, Kim TS: Development of a polymerase chain reaction applicable to rapid and sensitive detection of Clonorchis sinensis eggs in human stool samples. Pathog Glob Health 2013, 107:253-259.

doi:10.1186/1756-3305-6-314

Cite this article as: Qian et al:: Accuracy of the Kato-Katz method and formalin-ether concentration technique for the diagnosis of Clonorchis sinensis, and implication for assessing drug efficacy. Parasites \& Vectors 2013 6:314.

\section{Submit your next manuscript to BioMed Central and take full advantage of:}

- Convenient online submission

- Thorough peer review

- No space constraints or color figure charges

- Immediate publication on acceptance

- Inclusion in PubMed, CAS, Scopus and Google Scholar

- Research which is freely available for redistribution

Submit your manuscript at www.biomedcentral.com/submit
C BioMed Central 